\title{
Status and Plan of DUNE at Fermilab
}

\author{
Jaehoon Yu ${ }^{1}$ \\ The University of Texas at Arlington \\ Department of Physics, 502 Yates St., Arlington, TX 76019, USA \\ E-mail:jaehoonyu@uta.edu \\ On behalf of The DUNE Collaboration
}

Abstract: The discovery and the solid confirmation of neutrino flavor oscillation have been made throughout late 1990 to 2000s. Such oscillation is the clear proof of non-zero neutrino mass since this is caused by the fact that the neutrino mass eigenstates differ from that of the flavor. The neutrinos in the Standard Model, however, are massless leptons, and thus the neutrino flavor oscillation necessitates either the Standard Model to be significantly modified or a new theoretical paradigm to describe the universe better. In order to provide experimental information, it is essential for the properties of the neutrino oscillation be well understood through precision measurements of oscillation parameters, including the mixing angles, determination of the mass hierarchy between the three mass eigenstates and determination of the $\mathrm{CP}$ phase $\left(\delta_{\mathrm{CP}}\right)$ between neutrino and anti-neutrino oscillations. Among the several next generation neutrino experiments, this presentation covers the Deep Underground Neutrino Experiment (DUNE) which, with the high intensity neutrino beam facility, will help accomplish these goals. In this report, I will discuss the current status and plan of the DUNE experiment, including the results of the detector performance studies from the large scale prototype detectors operating at CERN and the timeline for construction and data taking of the experiment, as well as its physics prospects.

The 21st international workshop on neutrinos from accelerators (NuFact2019) August 26 - August 31, 2019

Daegu, Korea

\section{${ }^{1}$ Speaker}




\section{Introduction}

Three most representative discoveries relevant to the basic fundamental particle map of the Standard Model [1] in the past quarter century arguably are the observation of top quark at Fermilab announced in 1995 [2], the discovery of Higgs like scalar boson at CERN announced in 2012 [3] and neutrino flavor oscillation discovery which was confirmed throughout the '90s and the 2000's, reviewed in Ref. [4]. Of these three, the first confirms the Standard Model by completing the quark sector map, and the second, the newly discovered Higgs-like boson increasingly behaves like the one predicted by the Standard Model while the third goes against it. Since neutrino flavor oscillation occurs due to the fact that the flavor eigenstate differs from the mass eigenstate, the fact that the neutrinos undergo the flavor oscillation means that neutrinos have mass. The Standard Model (SM), however, does not prescribe masses to neutrinos, is broken in the neutrino sector and thus requires either a significant modification or a completely new paradigm of theory to fully describe nature.

To accomplish the important goal above, precise measurements of various neutrino oscillation parameters, such as the mixing angles and mass square differences, precise determination of the hierarchy between the three mass eigenstates and determination of the CP phase $\left(\delta_{\mathrm{CP}}\right)$ are essential. In addition, the detailed study of the CP violation in the neutrino sector in which the questions such as the neutrino and anti-neutrino symmetry is needed. These could lead to a new symmetry through the tension between theoretical predictions and experimental measurements.

In addition to the basic physics topic describe above, there are the lingering questions of the grand unification, the energy scale of the unification and the discovery of proton decay which had been sought for a long time. Understanding neutrinos of astrophysical origin, neutrinos from supernova burst and relic neutrinos as well as dark matter and other unknown particles still remains to be an essential question to be answered. Finally, understanding how the supernova and
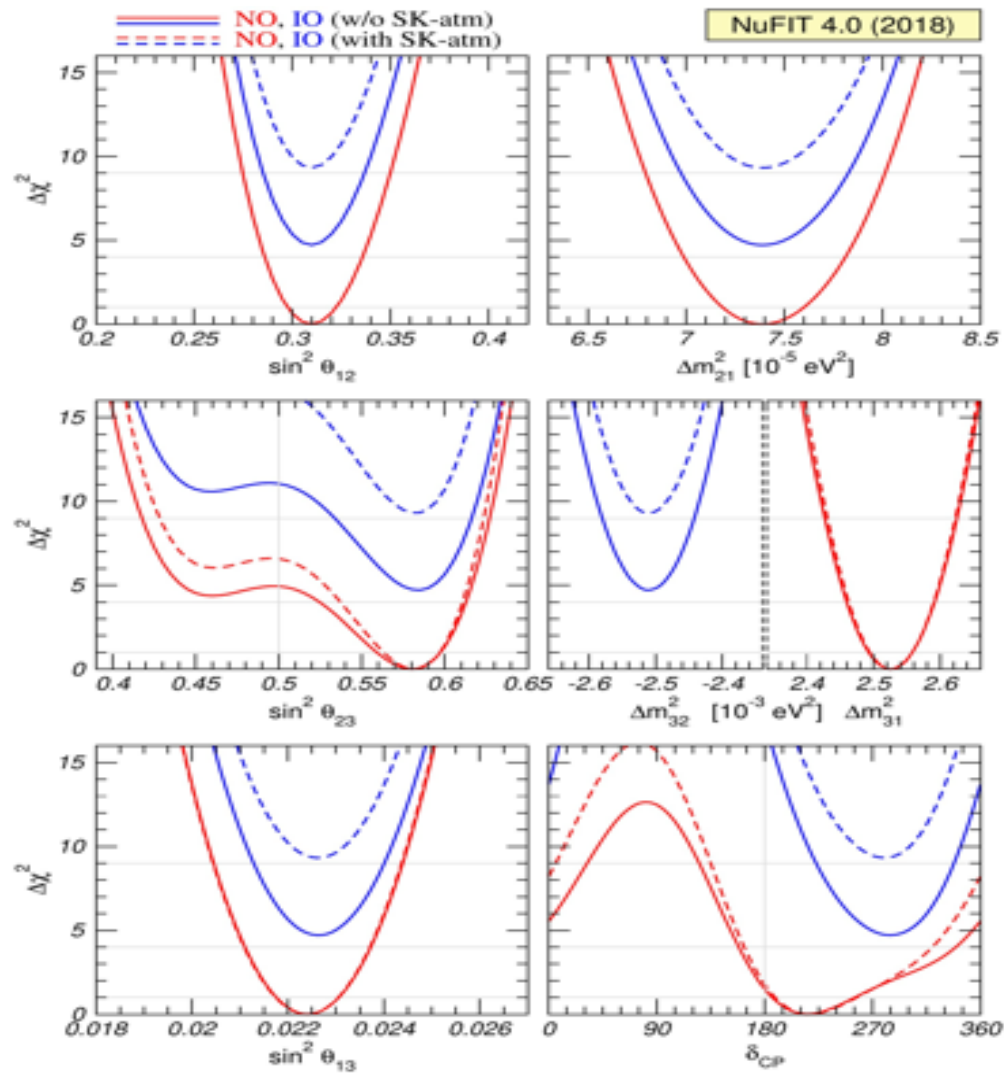

Figure. 1 Neutrino energy spectra measured in DUNE far detector through charged current interactions of (a) electron neutrinos, (b) electron anti-neutrinos, (c) muon neutrinos and (d) muon anti-neutrinos in normal mass ordering (NO) in red and inverted mass ordering (IO) in blue. 
the black hole can get formed, by measuring the flux of the electro-neutrinos coming from supernova explosion would be important and would provide additional information on neutrinos. Since neutrinos interact weakly, the experiments require high intensity neutrino beams as well as a large detector mass to obtain sufficient statistics. In addition, to help with reducing systematic uncertainties, the experiments need highly capable near and far detectors with a long baseline.

\section{Current Status of Neutrino Oscillation Parameter Measurements}

Many experiments have performed measurements of neutrino oscillation parameters. Figure 1 shows the current status of neutrino oscillation parameter measurements based on all available experimental data [5]. Figures $1(\mathrm{a})$ and $1(\mathrm{~b})$ show the $\sin ^{2} \theta_{12}$ and $\Delta \mathrm{M}_{23}^{2}$ measurements from atmospheric neutrino measurements of Super-Kamiokande (SK), IceCube, KM3NET, etc as well as long baseline experiment such as K2K, MINOS, Opera, NOvA, etc. Figures 1(c) and 1(d) show the $\sin ^{2} \theta_{23}$ and $\Delta \mathrm{M}^{2}{ }_{12}$ measurements from solar neutrino measurements of SNO, SK, Borexino, etc. and reactor result from KamLAND experiment.

Finally, Fig.1(e) and 1(f) are $\sin ^{2} \theta_{13}$ and $\delta_{\mathrm{CP}}$ measurement from long baseline experiments, such as MINOS, T2K, NOvA, etc and reactor experiments such as Daya Bay, RENO and Double Chooz. The red lines in the plots show the normal hierarchy (NH) while the blue lines show the inverted hierarchy (IH). Precision measurements will narrow the width of these curves and help determine even the mass hierarchy.

\section{Deep Underground Neutrino Experiment, Next Generation Neutrino Experiment}

High Energy Physics (HEP) community strategic planning documents in three regions, Europe, US and Japan, have concluded that precise understanding of neutrino oscillation is one of the top priorities. Following this strategy, the Deep Underground Neutrino Experiment (DUNE)[6] was born in the U.S. combining the U.S. and European experiments.

DUNE utilizes the high intensity neutrino beams sent from Fermi National Accelerator Laboratory (Fermilab) in Illinois, US, to the Sanford Underground Research Facility (SURF) in South Dakota, $1300 \mathrm{~km}$ away from the beam, $1500 \mathrm{~m}$ underground. DUNE is a joint effort of teams from all three regions, Europe, America and Asia. The collaboration consists of 1106 members of 84 institutions from 31 countries.

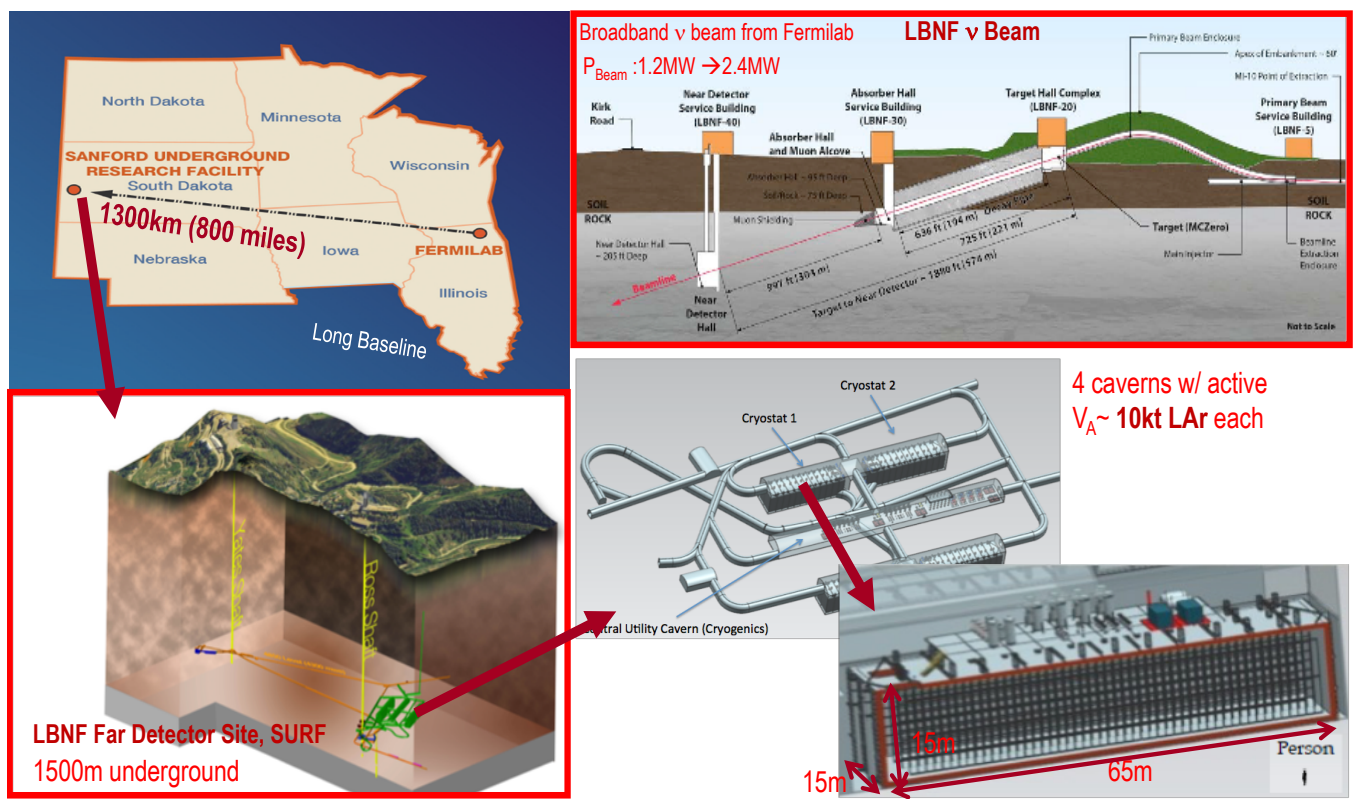

Figure. 2 Schematic diagrams of various component of DUNE. Top right shows the LBNF beam facility at Fermilab, top left shows the relative locations of the beam and the far site, bottom left shows the Sanford Underground Research Facility in Lead, SD, USA 1500m underground and bottom right shows the new area under excavation for the construction of the four caverns and a schematic diagram of one of the four 10kt LAr TPC detectors. 
As any long baseline neutrino experiment is, DUNE has two primary components, the Long Baseline Neutrino Facility (LBNF) which consists of high flux beam facility at Fermilab whose schematics is shown in top right of Fig. 2 and the far detector (FD) facility at SURF in South Dakota, USA, whose schematics is shown in top left, bottom left and bottom right of Fig. 2. The second component consists of the near detector (ND) to provide neutrino beam flux before the oscillation and the FD placed inside the FD cavern to measure the flux after the oscillation.

LBNF beam facility [7] is designed to provide a broadband neutrino beam with the beampower on day one expected to be $1.2 \mathrm{MW}$ and upgradable to $2.4 \mathrm{MW}$ proton beams whose energy is tunable in $60-120 \mathrm{GeV}$ range. Both the near and far detectors of DUNE are going to be onaxis to take advantage of the magnetic horn focused broadband neutrino beam optimized for Charge-Parity Violation studies by enabling access to two oscillation maxima.

The FD site is $1500 \mathrm{~m}$ underground in a gold mine which no longer operates. U.S. Department of Energy (DOE) has approved in late 2016 the construction of the new caverns that will host four liquid argon time projection chamber (LArTPC) detectors. The DUNE FD is a (TPC) of $40 \mathrm{kt}$ total active mass in a staged timeline, each of which has $10 \mathrm{kt}$ active mass as shown in the bottom right figure of Fig.2. The first two $10 \mathrm{kt}$ detectors will be constructed followed by the third $10 \mathrm{kt}$ about a year after start of the data taking of the first two and the fourth $10 \mathrm{kt}$ detector in a couple of years after the third. The technology for the first $10 \mathrm{kt}$ will be the single phase (SP) LArTPC while the that of the second $10 \mathrm{kt}$ detector will depend on the outcome of the ProtoDUNE dual phase (DP) experiment which is currently operating at CERN. The technology for the $3^{\text {rd }}$ module would either be an SP or DP, again depending on the outcome of the currently operating ProtoDUNE DP. The fourth detector technology is currently unknown but thanks to the large interests in the community, many $R \& D$ activities are ongoing for this.

The cavern excavation has been ongoing since the ground-breaking in 2017. Each of the four cryostats will have approximate inner dimension of $15 \mathrm{~m}(\mathrm{~W}) \times 15 \mathrm{~m}(\mathrm{H}) \times 65 \mathrm{~m}(\mathrm{~L})$, about the size of a large five story building. Since such large scale detectors have never been built, prototyping of the detector in both SP and DP technologies to provide information for technical design report (TDR) and for scalability testing will continue through the next few years.

The near detector (ND) [8] for DUNE will be located $570 \mathrm{~m}$ downstream of the neutrino target and will employ three detectors along the neutrino beam line. Figure 3 shows the current conceptual design of the DUNE ND complex which consists of three distinct detector technologies - LArTPC, high pressure gas TPC equipped with a magnet and an electromagnetic

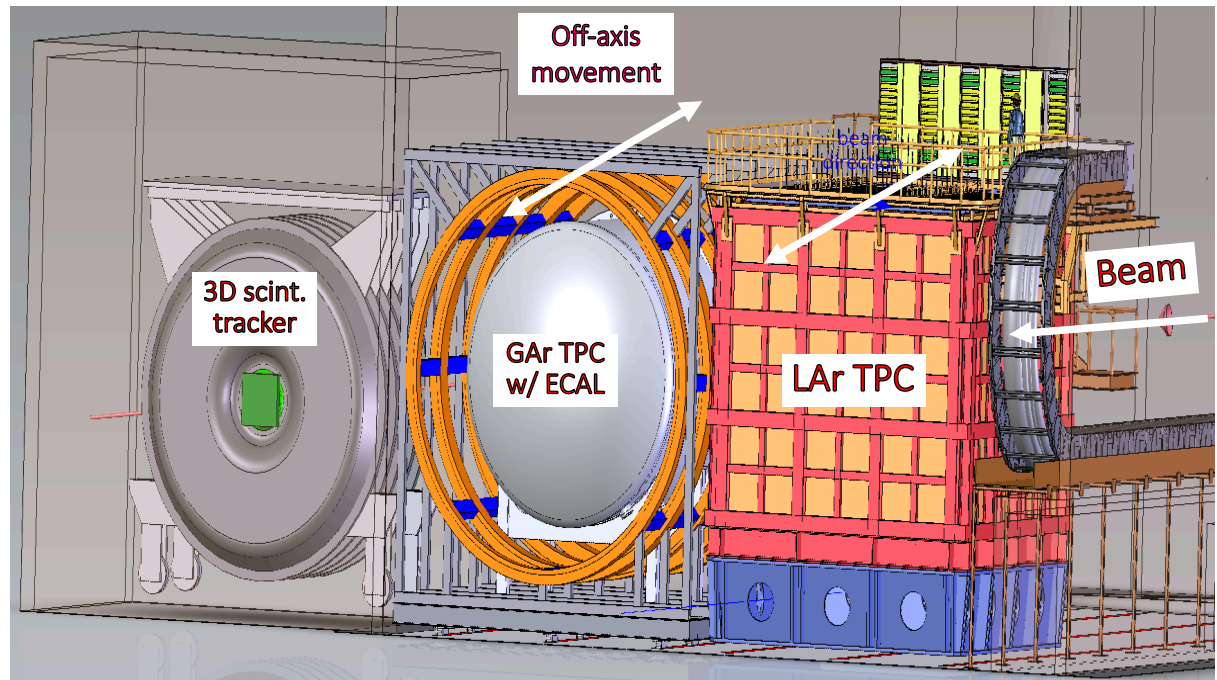

Figure. 3 DUNE Near Detector concept with three detectors along the beam line - LArTPC followed by high pressure gas TPC (HPGTPC) complemented with a magnet and EM calorimeter which then is followed by a 3D scintillation counter tracker. The LArTPC and the HPGTPC are movable perpendicular to the beam axis to enable off-axis flux measurements for systematic uncertainty reduction. 
calorimeter and the 3-D scintillation tracker. The ND hall is designed to accommodate the two upstream detectors to move horizontally, perpendicular to the neutrino beam axis (DUNE Prism), to allow off-axis neutrino flux measurements for reduction of systematic uncertainties.

The most upstream ND will be the $150 \mathrm{t}$ active mass LArTPC detector, the same technology as the far detectors to reduce the systematic uncertainty and will measure $v$-Ar interaction cross section high statistical precision, given the beam intensity. It will employ a modular design with dimension $0.7 \mathrm{mx} 0.7 \mathrm{mx} 1.8 \mathrm{~m}$. A beam testing of a smaller scale detector with four modules is planned in order to explore the performance of the modular concept. The high-pressure gas TPC detector that immediately follows the LArTPC will be able to detect $v$-Ar interactions with precision low threshold tracking, and the magnetization will allow precise muon momentum measurements which further assists the reduction of the neutrino flux systematic uncertainty. Finally, the 3D scintillation counter tracking system with $8 \mathrm{t}$ of active mass will provide fast onaxis beam monitoring.

\section{DUNE Physics Reach}

With the capacities of DUNE provided in the previous section, what would be the expected physics reach? The plots in Fig. 4 show the neutrino energy spectra measured through their charged-current interactions at DUNE FD. Figure 4. (a) and (c) are the spectrum of the electron neutrinos resulting from muon neutrino oscillation and that of the muon neutrinos which clearly shows depression at the peak of the electron neutrino spectrum from their disappearance. Similarly, Figure 4. (b) and (d) show the corresponding spectra in anti-neutrino running mode, assuming 3.5 year running each of neutrino and anti-neutrino modes for a staged scenario for the 70 kt-year of data collection [9].

By comparing the measured neutrino flux in FD in either the depression or appearance in these neutrino energy spectra to the un-oscillated neutrino flux measured at ND, the oscillation parameters can be measured precisely. The higher the statistics the more precise the measurements become in terms of its statistical uncertainties. In order to obtain more precise measurements beyond that of the statistical uncertainly, however, it is critical to have a good systematic understanding of the neutrino flux before the oscillation. The un-oscillated neutrino flux can be determined by varying the combination of powerful ND complex described previously.

Fig. 5 (a) shows the sensitivity for determining the CP phase $\left(\delta_{\mathrm{CP}}\right)$ with a staged approach for two $10 \mathrm{kt}$ active mass followed by additional two with a few year separation in between for 7 year
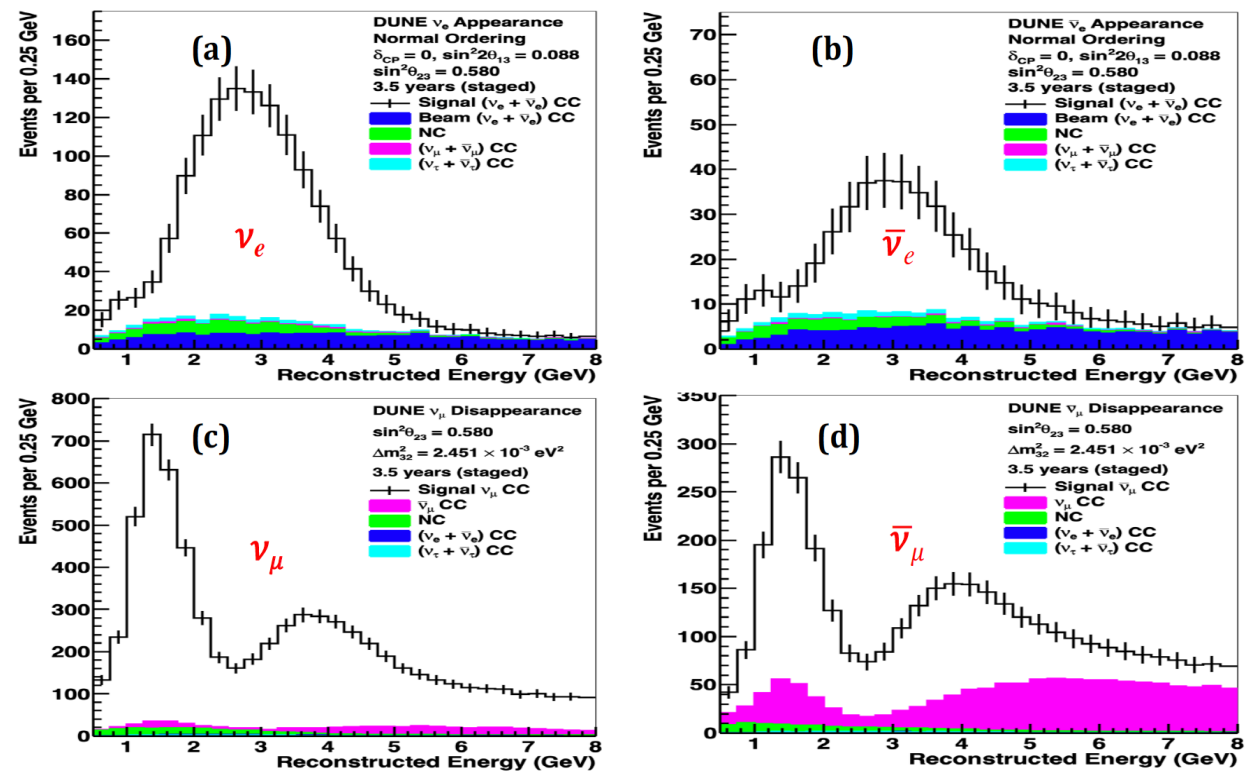

Figure. 4 Neutrino energy spectra measured in DUNE far detector through charged current interactions of (a) electron neutrinos, (b) electron anti-neutrinos, (c) muon neutrinos and (d) muon anti-neutrinos in normal mass hierarchy 

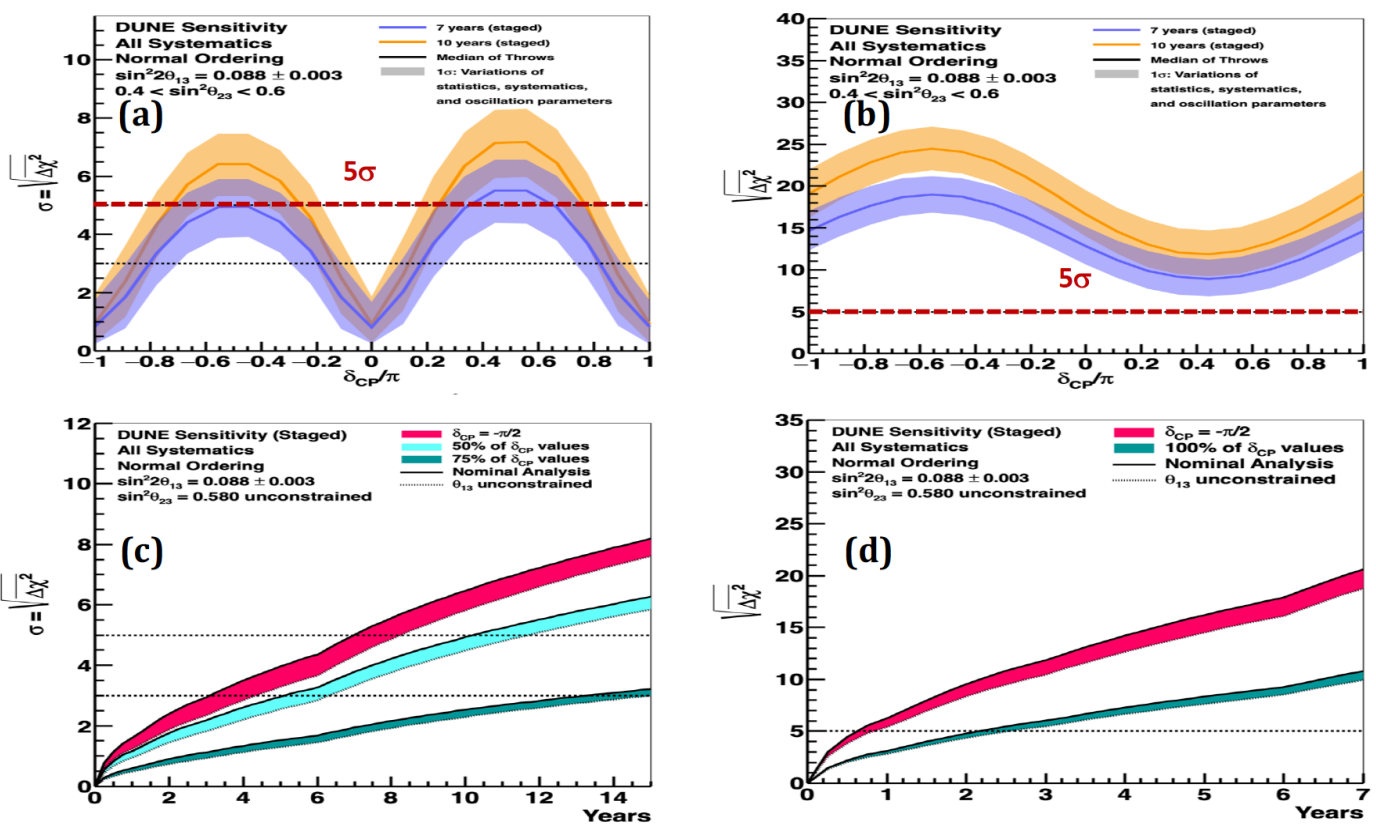

Figure. 5 Exclusion plots for $\delta_{C P}$ (a) for 7 year (green) and 10 year (orange) data taking with a staged active mass scenario, (b) the significance in mass ordering determination as a function of $\delta_{\mathrm{CP}} / \pi$ which depicts that mass ordering will be determined over $5 \sigma$ significance after 7 years of data taking (c) of $\delta_{C P}$ significance as a function of year in three different $\delta_{C P}$ scenarios (d) mass ordering significance as a function of year in two different $\delta_{C P}$ scenarios.

(green) and for 10 year (orange) running in normal mass ordering scenario. The band in Fig.5 (a) reflects the current uncertainties in $\theta_{13}$ and $\theta_{23}$ measurements [5] as indicated in the figure. The two dashed horizontal lines in both the plots show $3 \sigma$ and $5 \sigma$ significance. These show that a wide range of $\delta_{\mathrm{CP}}$ can be covered in each of the experiments with the potential for discovery beyond $5 \sigma$ level in about $0.2 \pi<\delta_{\mathrm{CP}}<0.8 \pi$ range. It important to point out that expected resolution of $\delta_{\mathrm{CP}}$ is of the order 10 degrees at $\delta_{\mathrm{CP}}=0$ for DUNE.

Figure 5.(b) shows the significance of mass ordering determination as a function of $\delta_{\mathrm{CP}}$ with staged approach for two 10kt active mass followed by additional two with a few year separation in between for 7 year (green) and for 10 year (orange) running, clearly demonstrating over $5 \sigma$ level significance of mass ordering determination for all range of $\delta_{\mathrm{CP}}$ after 7 years of data taking. Figures 5(c) and (d) show the significances for determining the CP phase $\left(\delta_{\mathrm{CP}}\right)$ and mass ordering as a function of the number of years of data taking. These clearly demonstrates that DUNE will be able to make statistically significant statements even before reaching the 10 year data taking.

More exciting potential is the possibility for observing supernova burst. It is expected that when a supernova explosion happens, the flux of the electron-neutrinos generated from the burst depends on the elapsed time from the time of burst. Figure 6(a) shows the measured electron as a function of time from the burst in seconds in DUNE FD through the $v_{e}+{ }^{40} \mathrm{Ar} \rightarrow e^{-}+{ }^{40} K^{*}$ reaction. Figure 6(a) demonstrates that the time dependence of the electron neutrino flux differs depending on the process of the burst and collapse into a black hole, especially in $0-0.25$ seconds after the burst, enabling the experiment to explore in detail the model for black hole formation in real time. In addition, the electron neutrino flux in $0.02-0.05$ second range differ dramatically between no-oscillation (blue), oscillation with the normal mass ordering (red) and oscillation with the inverted mass ordering (green). Therefore, precise measurement of electron neutrino flux as a function of elapsed time from the supernova burst enables clear determination of mass ordering.

Finally, thanks to high intensity proton beams and the large mass highly capable detectors, physics beyond the Standard Model (BSM) can be pursued in DUNE. In particular, since DUNE is LArTPC detector that can provide precision measurements of timing, energy and tracks together 

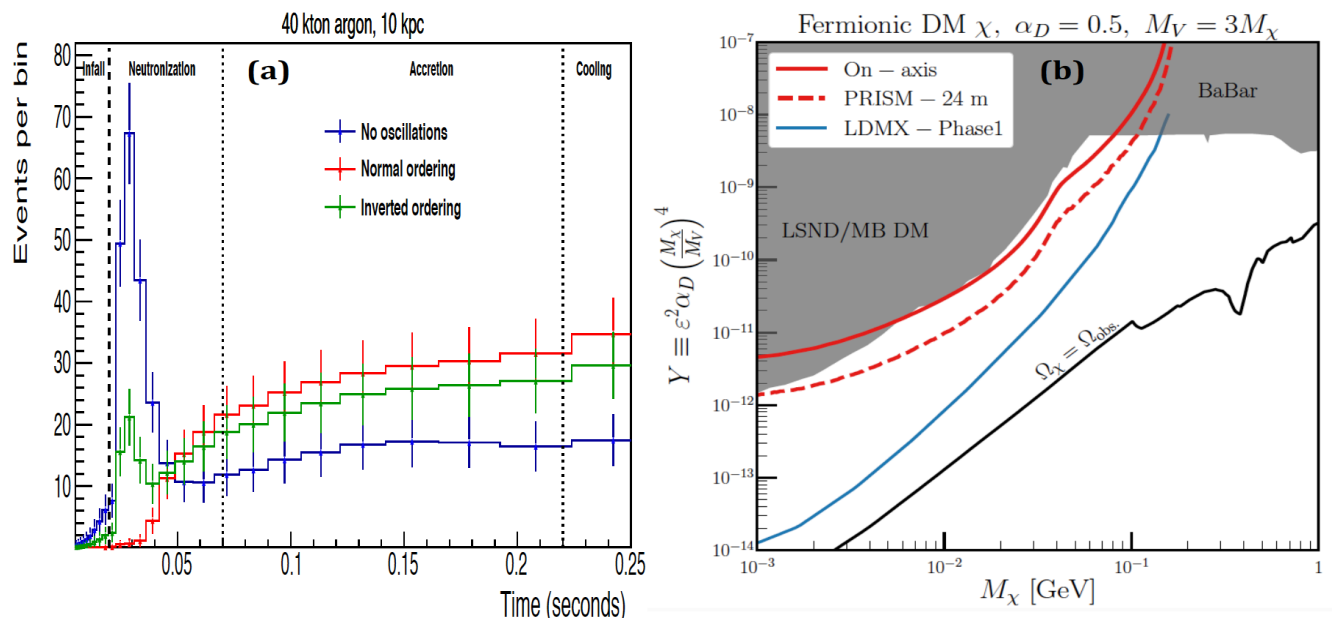

Figure. 6 (a) Electron neutrino flux as a function of time in seconds from the DUNE far detector for two different mass ordering scenarios and no oscillations (b) Low mass dark matter search sensitivity at the DUNE near detector

with low energy detection threshold, many BSM topics [10] can be explored in the experiment. Among these, DUNE can perform searches for low-mass dark matter (LDM) created in the beam and the boosted dark matter of cosmogenic origin. LDM could be produced in the neutrino target through a kinematic coupling [11] and detected in DUNE via elastic scattering resulting in a knockoff electron or a nuclear recoil, just like neutrinos. Since LDM particles are expected to be heavier than neutrinos, their average arrival time to ND is later than that of the neutrinos, providing a discriminating tool.

Furthermore, we have learned that the DUNE Prism can help improving the sensitivity for LDM search since as the LArTPC ND moves off-axis, the neutrino flux drops much faster than that of LDM and reduced background from neutrino interactions. Figure 6(b) shows the kinematic mixing parameter for LDM with respect to the LDM mass. The solid red line is the DUNE sensitivity on axis whereas the dashed line shows utilizing DUNE Prism up to $24 \mathrm{~m}$ off-axis in $6 \mathrm{~m}$ intervals at equal data taking time, after the staged 7 year data taking [12]. The plot clearly demonstrates that DUNE will make a significant contribution in search of dark matter especially in the mass range below $1 \mathrm{GeV}$ in which direct search experiments have a hard-wall to break through due to intrinsic radiological background in the detector.

In addition, many other BSM topics, such as searches for sterile neutrinos, non-standard interactions, boosted dark matter and heavy neutral leptons are feasible. An interesting opportunity for early physics at DUNE is the search for booted dark matter in its inelastic scattering using ProtoDUNE detectors [13]. The BSM opportunities are the area of physics which promotes strong collaboration between experimentalists and phenomenologists.

\section{Prototype Detectors}

Since it is necessary to construct prototypes prior to building large-scale experiments like DUNE, DUNE built two prototype detectors at CERN neutrino platform, one each for single and dualphased technologies in through 2018 and 2019. In a single-phase detector, the interaction occurs inside the liquid, and the ionization electrons from the traversing charged particles resulting from the interaction drift horizontally and get detected by the anode wire plane in the liquid. The active volume of the SP ProtoDUNE detector (ProtoDUNE SP) is $6.9 \mathrm{~m}(\mathrm{~W}) \times 6 \mathrm{~m}(\mathrm{H}) \times 7.2 \mathrm{~m}(\mathrm{~L})$ split into two $6.9 \mathrm{~m}(\mathrm{~W}) \times 6 \mathrm{~m}(\mathrm{H}) \times 3.6 \mathrm{~m}(\mathrm{~L})$ modules that share the single cathode plane in the middle. In the DP ProtoDUNE (ProtoDUNE DP), the interaction occurs in LAr but the ionization electrons drift vertically upward to the liquid-gas interface, extracted from liquid into the gas and amplified through the Large Electron Multiplier (LEM) before the charges get collected in gas. The active volume of ProtoDUNE DP is $6 \mathrm{~m}(\mathrm{~W}) \times 6 \mathrm{~m}(\mathrm{H}) \times 6 \mathrm{~m}(\mathrm{~L})$.

At the time of this presentation, ProtoDUNE SP took beam data from Sept. 2018 through the start of the long shutdown of CERN SPS in Nov. 2018. It has been taking cosmic ray data and 

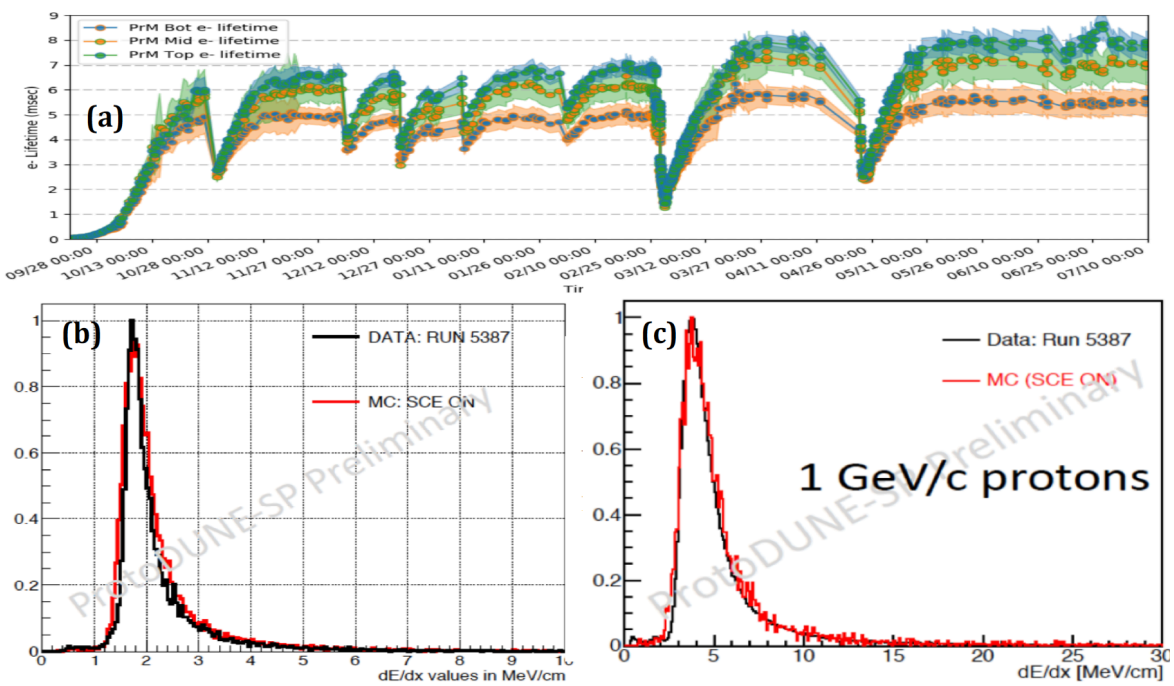

Figure. 7 (a) Electron lifetime which measures the LAr purity as a function of dates from turn on in ProtoDUNE SP (b) Space charge correction obtained from comparing MC to cosmic ray data (c) Proton response after the application of the space charge correction.

performing engineering tests throughout the past year. The analyses of the data taken through the past year and a half shows that high LAr purity to the level of $6 \mathrm{~ms}$ or higher drift electron lifetime can readily be accomplished, as shown in Fig. 7(a), the lifetime as a function of dates. The plot also shows that once an initial running condition was accomplished, the $6 \mathrm{~ms}$ or better purity can be reached within a short time interval. Figure 7(b) shows the space charge correction obtained from the comparisons of the cosmic data to the simulation. Figure 7(c) shows the detector response to beam protons after the space charge correction is applied, clearly demonstrating that the correction is effective and giving confidence in our understanding of the detector [14].

Proto-DUNE DP installation completed in June 2019. The detector was filled with LAr to its operational level. At the time of this presentation, the detector commissioning was ongoing. An acceptable operational stability of the charge readout plane which operates in gaseous argon at the top of the detector has been reached, and cosmic tracks and nuclear interactions have been seen in the detector. An important fact in these prototypes is that most of the components used in construction are the same size to be used in DUNE the final detectors, so ProtoDUNE detectors are almost completely scalable to the final DUNE detector.

\section{DUNE Financial Support}

For large scale experiments like Hyper-Kamiokande and DUNE, securing financial support is an essential aspect of the project. As was pointed out in previous sections, the cavern construction at the has been fully approved by the U.S. Department of Energy, the hosting agency and the excavation process has begun with a support over $\$ 100 \mathrm{M}$ level. And then support for LBNF neutrino beam facility which covers the proton beam, the neutrino target, the secondary beam focus, decay chain for neutrino beam generation and the beam dump is expected in a couple of years. Independently, the proton improvement plan necessary for high intensity proton beam for 1.2 MW beam power is strongly supported separately from LBNF by the U.S. government who provides over $\$ 100 \mathrm{M} /$ year support overall for DUNE.

Detector construction for prototyping also is in progress at CERN. CERN is an essential partner in DUNE. It has constructed neutrino platform in the north area of the CERN beam test area in the Previssin site on the French side. It has built two cryostats for ProtoDUNE and is committed to build the cryostat for the first 10kt DUNE detector out of the four. At the time of this presentation, the construction schedule for the first cryostat by GTT, the company that builds these cryostats, is being finalized as part of the overall scheduling of the DUNE project. CERN continues to play an important leadership role in the installation and operation of the prototype detectors.

Finally, U.K. has agreed in September 2017, to award \$88M project grant for UK institutions 
on DUNE. In addition, many other countries, such as France, Switzerland, Italy, Brazil, Korea, are making various contributions at different stages of the experiment. The commitment of the support DUNE currently has garnered is over several hundred million dollars.

\section{DUNE Timeline}

DUNE cavern excavation is ongoing and will be completed by 2021. DUNE has completed its technical design report [9] which was under review by the Fermilab Long Baseline Neutrino Committee (LBNC) at the time of this presentation. The second ProtoDUNE SP is planned and will be constructed through 2022 since the TDR design has modifications from the initial design. The ProtoDUNE DP is in commissioning and shows operational stability, while there still is a large amount of work left to definitively demonstrate the maturity of the technology to be used in the second 10kt FD module.

The construction of DUNE FD is expected to begin late 2021 for production of the components, and the installation of the first two $10 \mathrm{kt}$ active mass detectors in the cryostat is expected to complete by May 2026, following the completion of the cavern excavation and the construction of the two cryostats. DUNE FD cryostats are expected to be filled with LAr in 2025 -2026 , enabling the start of cosmic ray data taking in 2026 with beam ready in 2027 . The beam will become available with the $1.2 \mathrm{MW}$ power in $2026-2027$ time scale. This timeline and various milestones, including that of the LBNF will be finalized after the international project baseline exercise expected in spring 2020.

Finally, the DUNE ND which will be located about 570m downstream of the neutrino target will have its conceptual design report completed late 2019 or early 2020 . This will then be followed by its own prototyping and beam tests which will provide valuable information for its own technical design report in time for beam ready in 2027.

\section{Conclusions}

The neutrino sector and the Standard Model needs to be modified thanks to the solid experimental proof of neutrino flavor oscillation. Precise understanding of neutrino oscillation phenomena, precision measurements of the oscillation parameters and determining CP violation phase in lepton sector are essential for this. DUNE has been garnering strong financial supports and commitments from U.S.A., U.K., CERN and other partner countries and been making excellent progress. The far site cavern construction in South Dakota is fully funded and has been ongoing. Two large-scalable DUNE LArTPC prototype detectors have been operating and showing excellent performance. DUNE completed its FD TDR which is currently under review. ND CDR, prototyping and TDR will follow. The construction schedule for DUNE targets beam data in 2027 but will be more precisely determined after the project baselining in spring 2020 .

\section{References}

[1] S. L. Glashow, Nucl. Phys. 22, 579 (1961); S. Weinberg, Phys. Rev. Lett. 19, 1264 (1967)

[2] F. Abe et al, CDF Collaboration, Phys. Rev. Lett. 74, 2626 (1995); S. Abachi et al., DZero Collaboration, Phys, Rev. Lett. 74, 2422 (1995)

[3] ATLAS Collab., Phys. Lett. B716, 1 (2012); CMS Collab., Phys. Lett. B716, 30 (2012)

[4] V.Barger, D.Marfatia, K.L.Whinsnant, "The Physics of Neutrinos," Princeton University Press,. ISBN 978-0-691-12853-5 (2012)

[5] I.Esteban, M.C. Gonzalez-Farcia, A.Hernandez-Cabezudo, M.Maltoni, T.Schwetz, J.HEP 01, 106 (2019); arXiv:1811.05487 (2018) NuFit4.0

[6] Deep Underground Neutrino Experiment, http://www.dunescience.org/

[7] C. Densham in these proceedings

[8] A. Bross and S. Kim in these proceedings

[9] DUNE Technical Design Report (TDR) under LBNC review; R. Patterson's Fermilab JETP Seminar, http://vmsstreamer1.fnal.gov/Lectures/WC/presentations/190802Patterson.pdf (2019)

$[10] \mathrm{K}$. Cho in these proceedings

[11] B. Batell, M. Pospelov, and A. Ritz, Phys. Rev. D80, 095024 (2009)

[12] V. De Romeri, K.J. Kelly and P.A.N. Machado, Phys. Rev. D100, 095010 (2019)

[13] A.Chatterjee at al., Phys. Rev. D98, 075027 (2018)

[14] J. Bian and J. Thompson in these proceedings 